\title{
Estudio teórico del proceso de tutoría psicopedagógica en la educación superior
}

Theoretical study of the process of psychopedagogical tutoring in higher education

\author{
MSc, Pablo Parra Silva ${ }^{1}$ \\ pparra@uteq.edu.ec \\ MSc, Badie Cerezo Segovia \\ MSc, Daniel Parra Gavilanes ${ }^{1}$ \\ dparra@uteq.edu.ec \\ MSc, Mirella Flores Jaén ${ }^{1}$ \\ mflores@uteq.edu.ec
}

Recibido: 1/04/2018, Aceptado: 1/06/2018

\begin{abstract}
RESUMEN
Contribución teórica a la formación profesional, en un escenario de innovación educativa, donde la tutoría psicopedagógica tiene distinción excepcional. El objeto de estudio teórico es contribución a la epistemología del proceso de tutoría psicopedagógica en la educación superior. El objetivo es conocer lo que implica el proceso tutorial, desde una perspectiva psicopedagógica; preguntándose respecto a: Precisiones teóricas del concepto de tutorías universitarias; función tutorial psicopedagógica en la formación universitaria; bases teóricas que estructuran la epistemología del proceso tutorial psicopedagógico; contribución de la epistemología del proceso de tutorías psicopedagógicas, a la comprensión teórica y precisión científica del tema; tendencias teóricas del proceso de tutoría psicopedagógica en la educación superior. En la Universidad Técnica Estatal de Quevedo-Ecuador (UTEQ), la urgente necesidad de evaluar, analizar, corregir y mejorar, a través de la construcción del plan de acción tutorial, que actualice, innove y alcance niveles de excelencia académica su proceso educativo.
\end{abstract}

Palabras clave: Proceso, tutoría psicopedagógica, epistemología, tendencias

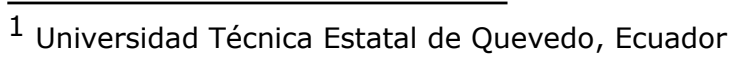

Revista científica Ciencia y Tecnología Vol 18 No 19 págs. 13-23

http://cienciaytecnologia.uteg.edu.ec 


\begin{abstract}
Theoretical contribution to professional training, in a scenario of educational innovation, where psychopedagogical tutoring has exceptional distinction. The object of theoretical study is contribution to the epistemology of the process of psychopedagogical tutoring in higher education. The objective is to know what the tutorial process implies, from a psychopedagogical perspective; asking about: Theoretical details of the concept of university tutorials; psychopedagogical tutorial function in university education; theoretical bases that structure the epistemology of the psychopedagogical tutorial process; contribution of the epistemology of the process of psychopedagogical tutorials, to the theoretical understanding and scientific precision of the subject; Theoretical tendencies of the psychopedagogical tutoring process in higher education. At the State Technical University of QuevedoEcuador (UTEQ), the urgent need to evaluate, analyze, correct and improve, through the construction of the tutorial action plan, to update, innovate and reach levels of academic excellence in the educational process.
\end{abstract}

Keywords: Process, psychopedagogical tutoring, epistemology, trends

\title{
Introducción
}

El proceso de tutoría psicopedagógica es trascendente en el escenario de innovación educativa, donde la tutoría psicopedagógica tiene una distinción excepcional (González Tirados y González Maura), p. 26. Exige conocimientos y preparación académica, profesional y axiológica por parte del docente; como también adaptación, participación y protagonismo del estudiante en su proceso de formación integral. "La figura del profesor- tutor adquiere relevancia en la Universidad actual en la que la formación integral del profesional constituye un objetivo esencial" (González Maura, 2006, p. 23). El objeto de estudio es el fortalecimiento de la epistemología del proceso de tutoría psicopedagógica en la educación superior en la UTEQ. Si se pretende aplicar un modelo tutorial de excelencia, se debe fundamentar desde la gnoseología, para comprender realmente el alcance y dimensión de esta singular tarea, adicionando criterios axiológicos y de responsabilidad compartida.

\section{Desarrollo}

Precisiones teóricas sobre función tutorial psicopedagógica. Ordaz (2011) define la palabra tutor, tomando su origen en el latín "tuteläris": el que guía, ampara, protege o defiende (Ordaz, 2011, p. 27). El término fue introducido en los sistemas de educación a distancia por Open University de Inglaterra. Tutelar, es el que protege o ampara. Es un agente educativo, un profesional que intencionalmente promueve, facilita y mantiene la retroalimentación y la asesoraría académica y no académica, para apoyar la creación de condiciones que favorezcan la calidad del aprendizaje y la realización personal y profesional (Pí et al., 2011, p. 2). La tutoría se define como el acompañamiento y apoyo docente de carácter individual, basada en una atención personalizada que favorece una mejor comprensión de los problemas que enfrenta el alumno, por parte del profesor, en lo que se refiere a su 


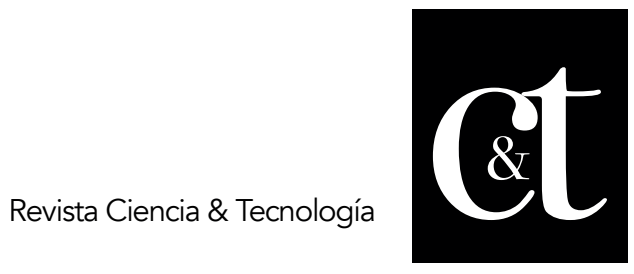

No. 19, 31 de julio de 2018

ISSN impreso: 1390 - 6321

adaptación al ambiente universitario, a las condiciones individuales para un desempeño aceptable durante su formación y para el logro de los objetivos académicos que le permitirán enfrentar los compromisos de su futura práctica profesional.

La misión primordial de dicha tutoría es proveer orientación sistemática al estudiante, desplegada a lo largo del proceso formativo; desarrollar una gran capacidad para enriquecer la práctica educativa y estimular las potencialidades para el aprendizaje y el desempeño profesional de sus actores: los profesores y los alumnos" (Tejada Tayabas y Arias Galicia, 2006, p. 25). El carácter personal e individualizado, como un acompañamiento comprensivo, son enfocados por Bravo y Sánchez (2008), que citando a B. Collazo, dicen que la tutoría en el Modelo Pedagógico por Competencias de la UTEQ, es "el proceso que se da entre tutor y estudiante con una responsabilidad compartida por ambos (...), donde se logre un Modelo de Tutoría Integral: una Tutoría Educativa Orientadora y Personalizada, que contempla lo académico, lo vocacional-profesional, lo personológico y lo investigativo.

La finalidad es potenciar el desarrollo integral pleno y cada vez más independiente de los estudiantes, como parte de su preparación para la vida. Aprender a: conocer; a hacer; a ser y convivir. Aprender a Aprender" (Bravo y Sánchez, 2008, p.3). Los autores, además sostienen, que la tutoría es una actividad dirigida a la formación integral del estudiante, que se realiza paralelamente con el proceso de enseñanzaaprendizaje y que requiere de una atención individualizada, pero sobre todo de una formación especializada del profesor. Esa formación especializada es precisamente la pedagogía universitaria, las fortalezas de metodología de investigación científica, la didáctica, la ética tutorial y profesional. Está vinculado a otros corolarios como los problemas de deserción, reprobación y baja titulación. Esto obedece a otros elementos significativos como: la baja calidad de los servicios educativos, deficientes condiciones de gestión académica y situaciones personales adversas de los/las estudiantes, que señalan como elementos causales a la desorganización, el retraimiento social y conductas disruptivas en su proceso de formación profesional. Se vislumbra a las tutorías, como acciones "más vinculadas a ser un sistema de acompañamiento y orientación eficiente que ofrece la institución, para que apoye a los estudiantes a mejorar su empeño, enseñándoles a pensar, así como eliminar los actuales problemas de deserción, rezago escolar y con ello elevar la eficiencia terminal" (Ordaz, 2011).

Epistemología del proceso tutorial psicopedagógico en la educación superior. Para el 2018, se pregona un cambio sustantivo en los paradigmas universitarios, en lo referente al aprendizaje, al trabajo colaborativo y a las tareas autónomas de los/las estudiantes. Pero, tomando como referencia lo acontecido en estos últimos ocho años, donde la UTEQ, adoptó el modelo educativo por competencias y, por tanto, las tutorías se convirtieron en una nueva actitud de parte del profesor, para orientar, guiar y fortalecer sus funciones, buscando encontrar una vinculación de próspera y eficiente utilidad; se encuentran variadas limitaciones, que aún no han podido ser 


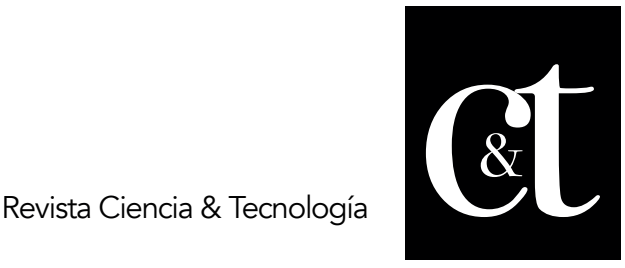

No. 19, 31 de julio de 2018

ISSN impreso: 1390 - 6321

superadas.

La tarea conjunta entre docente y estudiante es crear un clima favorable en los diferentes contextos, para lograr modos de actuación adecuados, en correspondencia con el perfil profesional de la Carrera. Este es un pilar importante que favorece la integración de los tutorados y su compromiso con el proceso de formación, para prevenir la deserción. Ojalvo (2005), menciona: "La tutoría es un proceso de acompañamiento durante la formación de los estudiantes, que se concreta mediante la atención personalizada a un alumno o a un grupo reducido de alumnos por parte de académicos competentes y formados para esta función, apoyándose conceptualmente en las teorías del aprendizaje más que en las de enseñanza. Dicho proceso de acompañamiento que comprende un conjunto sistematizado de acciones educativas centradas en el estudiante, está orientado básicamente a mejorar su rendimiento académico" (Ojalvo, 2005, p. 14).

Gimeno Sacristán, referido por Baudelio Lara García (2002), "señala que una de las dificultades para ofrecer una definición válida de currículum que sea universalmente aceptada, estriba en que un concepto se define dentro de un esquema de conocimiento (...), la tutoría académica, depende de marcos de interpretación muy variables. (...) El significado concreto del concepto de tutoría académica depende de su inserción en un determinado nivel educativo y de los propósitos generales que tiene ese nivel dentro del sistema escolar y dentro del proceso de escolarización. (...) Su significado específico va a variar en función de los propósitos que se persiguen en el proceso de formación en cada nivel o programa" (Lara García, 2002, p. 3).

Sin embargo, uno de los aspectos más controversiales que plantean Bravo y Sánchez (2008) en su enfoque sobre el Modelo por Competencias, son las características personales y profesionales que debe mostrar el tutor: tener una personalidad equilibrada, ser sensible para captar y entender los problemas juveniles, tener capacidad para entablar relaciones afectuosas y cordiales con los demás, lograr preparación académica en el área del conocimiento, tener conocimientos teóricos sobre educación y ciencias afines, manejar las relaciones interpersonales direccionándolas adecuadamente y con motivación, a su vez un conocimiento claro sobre técnicas de diagnóstico e intervención educativa.

Ojalvo (2005) resume que las principales funciones a desarrollar por los tutores son: "Conocer intereses y aptitudes de los alumnos, dirigir su proceso educativo, personalizar los procesos de enseñanza aprendizaje, lograr integración de estudiantes a sus grupos y a la institución educativa, contribuir al desarrollo del grupo-clase, coordinar la actividad docente de los profesores que trabajan con un mismo grupo de alumnos a partir de sesiones de evaluación, propiciar clima de convivencia, promover tolerancia y cooperación dentro del aula, aunar el esfuerzo individual con el colectivo, dar seguimiento a los aprendizajes de los estudiantes, ayudar a la solución de problemas y dificultades individual o colectiva, brindar orientación académica, atender el desarrollo vocacional y profesional, desarrollar 


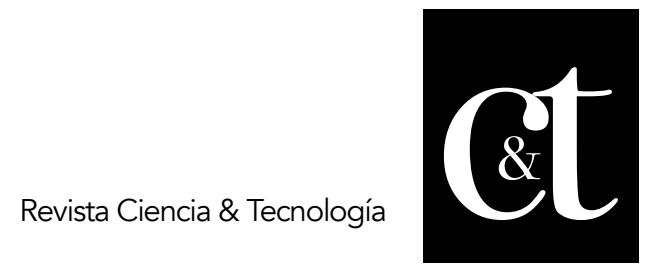

No. 19, 31 de julio de 2018

ISSN impreso: 1390 - 6321

actitudes y valores en los estudiantes vinculados a las normas de la institución y la sociedad" (Ojalvo, 2005, p. 17). Comprensión teórica y precisión científica del tema. El rol tutorial implica haber logrado determinado nivel de competencias.

Competencia cultural del conocimiento, donde el profesor domina la asignatura que imparte y tiene conocimientos vinculados con el campo de la especialización donde proporciona tales informaciones académicas. Manejar la competencia pedagógica, utilizando con certeza la didáctica para transmitir conocimientos y promover competencias, actitudes y valores. Significativa competencia de investigación, relacionada con la búsqueda, la curiosidad científica, la profundización de los conocimientos, la motivación por el descubrimiento, la ampliación coherente y objetiva de la información, la capacidad recreativa y dinámica de adquirir el conocimiento, así como la adecuada utilización de la tecnología para la actualización de todo tipo de informaciones. Por consecuencia, también está la competencia tecnológica, con el uso adecuado de herramientas, recursos y lenguajes informáticos, digitales, audiovisuales. Fundamentalmente, la competencia axiológica personal, que implica ser una persona asertiva con inteligencia emocional, autoestima, empatía, adecuadas relaciones interpersonales, buenas intenciones, honestidad y rigor científico a toda prueba.

Así mismo, para González Maura (2006), "el tutor juega un papel importante en el proyecto educativo, ya que apoya a los alumnos en actitudes, como las de crear en ellos la necesidad de capacitarse, de explorar aptitudes; de mejorar su aprendizaje y tomar conciencia, de manera responsable, de su futuro. La tarea del tutor, entonces, consiste en estimular las capacidades y procesos de pensamiento, de toma de decisiones y de resolución de problemas. Para ello, la tutoría debe ofrecerse a lo largo de los diferentes niveles de la universidad; vincular a las diversas instancias y personas que participan en el proceso educativo; atender a las características particulares de cada alumno; darse en términos de elevada confidencialidad y respeto; y, buscar que el alumno se responsabilice de su propio proceso de aprendizaje mediante la toma de conciencia de su libertad y de su compromiso con él y con los demás" (González Maura, 2006, p. 34). La tutoría pretende orientar y dar seguimiento al desarrollo de los estudiantes, lo mismo que apoyarles en los aspectos cognitivos y afectivos del aprendizaje.

Busca fomentar su capacidad crítica y creadora y su rendimiento académico, así como perfeccionar su evolución social y personal. "Dar seguimiento al desarrollo alcanzado por los alumnos, esto es llevar un registro de cada alumno tutorado, motivar y dar retroalimentación de los avances alcanzados y animarlos a seguir con su desarrollo personal, como académico como profesional" (Castro Cuesta, 2014, p. 126). La acción tutorial debe estar siempre atenta a la mejora de las circunstancias del aprendizaje y de canalizar al estudiante a las instancias en las que pueda recibir una atención especializada, con el propósito de resolver problemas que pueden interferir en su crecimiento intelectual y emocional, hecho que implica la interacción entre el tutor y el tutorado (Coriat Benarroch y Sanz Oro, 2009, p. 14). 


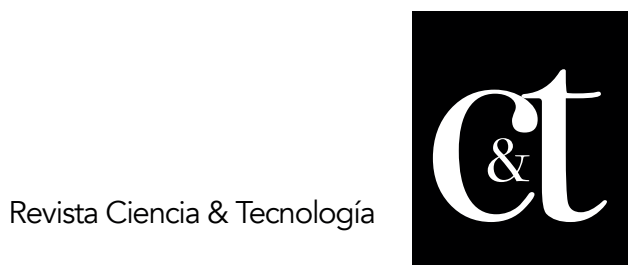

No. 19, 31 de julio de 2018

ISSN impreso: 1390 - 6321

Para apoyar la actividad tutorial y el desarrollo de los estudiantes, se requiere, además de la tutoría, de la interacción con otras entidades académicas y administrativas, como son los profesores de grupo o las academias de profesores (horizontales, departamentales, disciplinares o multidisciplinares); las unidades de atención médica y psicológica; programas de educación continua y extensión universitaria; instancias de orientación vocacional y programas de apoyo económico a los estudiantes; cursos de inducción a la universidad; cursos remediales; cursos para la adquisición y desarrollo de hábitos de estudio y trabajo; cursos para el desarrollo de habilidades. Los actores e instancias mencionadas tienen a su cargo la práctica docente (en el caso de los profesores) y un conjunto de actividades distintas a ésta y a la tutoría, que contribuyen y complementan a las dos primeras. Éstos deben constituir los programas para la mejora de la calidad del proceso educativo. Para Lobato (2004), "la realización de esta tutoría demanda la puesta en práctica de una intervención articulada en unas estrategias concretas y apoyada en una serie de recursos pertinentes en la consecución de los objetivos que dan sentido y significado a la acción tutorial". Pero, significativamente cita a Kuth (1993), quien en una investigación cualitativa y cuantitativa a estudiantes de doce universidades anglosajonas, sintetizaba cinco categorías de actividades fuera del aula: a) Competencia personal: autonomía, confianza en sí mismo, asertividad, clarificación de los propios objetivos en la vida, relación interpersonal ...; b) Competencia práctica: organización del tiempo, desenvolvimiento en aspectos burocráticos ...; c) Complejidad cognitiva: pensamiento crítico, capacidad para examinar distintos puntos de vista, aplicar y extrapolar aprendizajes en diferentes áreas, examinar diferentes perspectivas ...; d) Conocimientos y habilidades académicas: conocimientos adquiridos, capacidad de estudiar e investigar, manejo de diversas fuentes de información ...; $y, e$ ) Altruismo y sentido estético: sensibilidad hacia las necesidades de los demás, aprender a trabajar con personas distintas, aprecio del arte en general, de la naturaleza ..." (Lobato, 2004, p. 3).

\section{Principales tendencias}

El Plan de Acción Tutorial (PAT), constituirá ese documento sistemático de direccionamiento, que busca ajustarse como una propuesta lógica de acción tutorial en todos sus ámbitos. Se fundamenta en el principio de prevención, buscando anticiparse con oportunidad, en procura de superar o evitar posibles dificultades y conflictos, por lo que la tutoría deviene en una actividad proactiva y participativa permanente entre profesor y estudiante. Por otra parte, el principio de sistematización que representa un Plan de Acción Tutorial, debe considerar a todos los sujetos implicados en el proceso formativo profesionalizante. En el campo tutorial, las tecnologías de información y comunicación son herramientas fundamentales y junto con software y plataformas informáticas, aceleran el proceso y permiten un acercamiento significativo al estudiante. Siempre que se fundamente en principios axiológicos y en programaciones con resultados significativos para los involucrados. 
Métodos Realización de una investigación documental, con aportes significativos de investigadores, utilizando método inductivo-deductivo. Con base a estudio propuesto por los autores, en el año 2014; que mostraban un total de veintiocho parámetros evaluados, de los que se consideraron el enfoque específico de las tutorías. Una muestra de 462 estudiantes de la Carrera de Contabilidad, revela importantes aspectos relacionados con el rol tutorial.

\section{Resultados}

De esta acomodaticia perspectiva, que presenta las primeras apreciaciones del rol tutorial, dentro del modelo educativo por competencias que había iniciado la UTEQ, se toman a manera de ejemplo seis de aquellos indicadores:

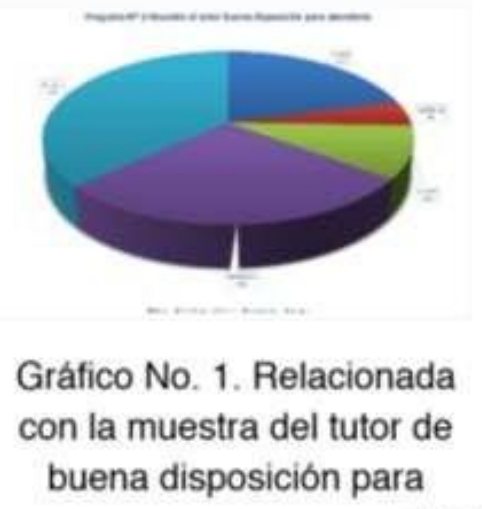

\begin{tabular}{|r|r|}
\hline Nunca & $21.00 \%$ \\
\hline $\begin{array}{c}\text { Casi } \\
\text { Nunca }\end{array}$ & $4.55 \%$ \\
\hline A veces & $9.74 \%$ \\
\hline $\begin{array}{c}\text { Casi } \\
\text { siempre }\end{array}$ & $27.92 \%$ \\
\hline Siempre & $36.80 \%$ \\
\hline \multicolumn{1}{|c|}{} & $100.00 \%$ \\
\hline
\end{tabular}

atender al estudiante.

Fuente: Página web institucional de la UTEQ. Año 2014.

Un docente-tutor debe estar dispuesto a atender al estudiante "siempre". Las demás variables señaladas en esta muestra, no hacen otra cosa que evidenciar el divorcio entre el rol del docente y el rol del tutor. Se exterioriza que el $21 \%$ de los docentes, "nunca" tuvieron la predisposición para atender al estudiante.

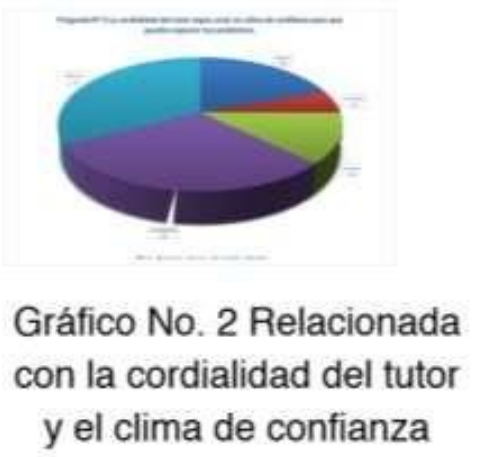

\begin{tabular}{|r|r|}
\hline Nunca & $19.70 \%$ \\
\hline $\begin{array}{c}\text { Casi } \\
\text { Nunca }\end{array}$ & $5.41 \%$ \\
\hline A veces & $12.12 \%$ \\
\hline $\begin{array}{c}\text { Casi } \\
\text { siempre }\end{array}$ & $30.09 \%$ \\
\hline Siempre & $32.68 \%$ \\
\hline & $100.00 \%$ \\
\hline
\end{tabular}

para exponer problemas.

Fuente: Página web institucional de la UTEQ. Año 2014. 
La encuesta plantea un parámetro muy parcial: la cordialidad del tutor, creando un clima de confianza para exteriorizar problemas por parte del estudiante. Sin embargo, el ambiente pleno para mostrar "siempre" dicha cordialidad, apenas alcanza el $32,68 \%$ de los momentos tutoriales. El casi $68 \%$, no hay tal cordialidad a plenitud, como debería constituir el encuentro tutorial sistematizado, como acciones planificadas, con resultados significativos para los involucrados. Además, resulta de enorme preocupación, que un $19,7 \%$ de las veces "nunca haya tal cordialidad".

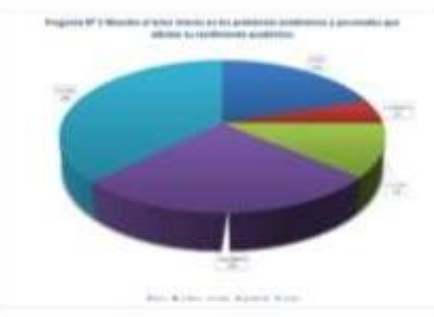

Gráfico No. 3. Relacionada con las muestras de interés del tutor hacia los problemas académicos y personales que afectan el rendimiento.

Un siguiente cuestionamiento enfoca las muestras de interés que desarrolla el tutor, en relación con los problemas académicos y personales que afectan el rendimiento. Vuelve a ocurrir, que el $19,91 \%$ de las ocasiones, "nunca" se preocupan los profesores por aquello. Entonces, habría que reparar en el hecho de saber, si los docentes de la UTEQ, se involucraron epistemológica y axiológicamente en la filosofía del modelo educativo que había adoptado la institución de educación superior.

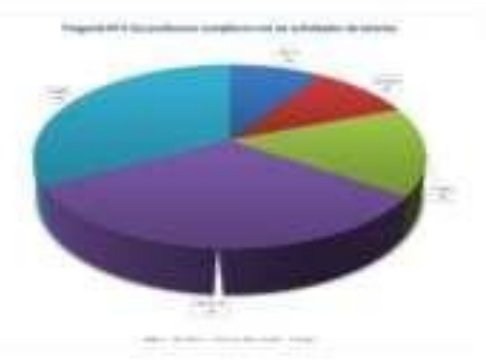

Gráfico No. 4 Relacionada con el cumplimiento de las actividades de tutoría por parte de los profesores. Fuente: Página web institucional de la UTEQ. Año 2014. 
Al estudiante se le inquiere respecto a si los docentes cumplieron con las actividades de tutorías. Siempre lo hicieron el 32,9\%. Entendemos, que la acción tutorial no puede ser cicateada ni desmerecida, para nadie. Un amplio 34,63\% a veces, casi nunca o nunca, pudieron reconocer el cumplimiento de las actividades de tutoría de los profesores. Sin embargo, a los docentes se les asignó una carga horaria y un costo económico para el cumplimiento de dichas actividades tutoriales desde aquel entonces.

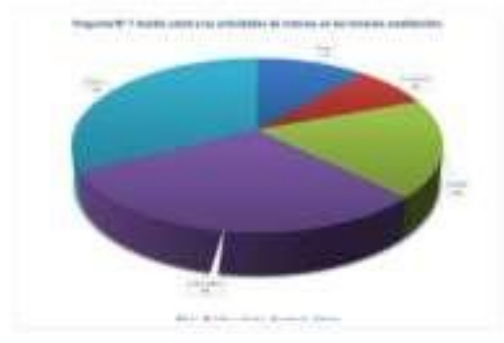

Gráfico No. 5. Relacionada con la asistencia del

\begin{tabular}{|c|c|}
\hline Nunca & $11.47 \%$ \\
\hline $\begin{array}{c}\text { Casi } \\
\text { Nunca }\end{array}$ & $7.79 \%$ \\
\hline A veces & $18.18 \%$ \\
\hline $\begin{array}{c}\text { Casi } \\
\text { siempre }\end{array}$ & $30.09 \%$ \\
\hline Siempre & $32.47 \%$ \\
\hline & $100.00 \%$ \\
\hline
\end{tabular}

estudiante en los horarios establecidos para las tutorias.

Reiterando lo anterior, tampoco el estudiante cumplió con los horarios establecidos para las tutorías. Mostrando, además, un desconocimiento y poco interés por la acción tutorial dentro de su formación integral. Un 63\%, siempre o casi siempre, cumplió con los horarios establecidos. El resto no.

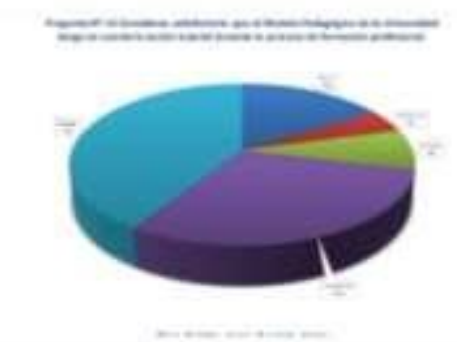

Gráfico No. 6. Relacionada la satisfacción de que el Modelo

\begin{tabular}{|c|c|}
\hline Nunca & $16.23 \%$ \\
\hline $\begin{array}{c}\text { Casi } \\
\text { Nunca }\end{array}$ & $4.55 \%$ \\
\hline A veces & $8.01 \%$ \\
\hline $\begin{array}{c}\text { Casi } \\
\text { siempre }\end{array}$ & $30.30 \%$ \\
\hline Siempre & $40.91 \%$ \\
\hline & $100.00 \%$ \\
\hline
\end{tabular}

Pedagógico de la Universidad, tenga en cuenta la acción tutorial durante el proceso de formación profesional. 
Finalmente, era de esperarse que en la búsqueda de conocer la relación entre del modelo pedagógico y la acción tutorial, un aproximado $29 \%$ no se encuentra satisfecho, en cuanto a los resultados de la acción del docente como tutor en este modelo educativo.

\section{Conclusiones}

La búsqueda de un enfoque teórico que propicie el fortalecimiento epistemológico de las tutorías psicopedagógicas universitarias, constituye un ingrediente de notable valor, para la comprensión adecuada del proceso educativo que se ha llevado a efecto en la UTEQ, en estos últimos ocho años que han transcurrido. Se busca propiciar una mejor perspectiva, mucho más cuando se lograron superar dos acreditaciones anteriores y se espera, para el año 2018, mantenerse en una categoría " $B$ " en el concierto universitario del Ecuador. Se avecinan, en consecuencia, urgentes avances teóricos en la línea de investigación educativa, que serán necesarios fortalecerlos de una manera minuciosa y observando una actitud crítica constructiva hacia el futuro de la institución de educación superior.

\section{Referencias bibliográficas}

Bravo, G., \& Sánchez, L. (2008). El profesor-tutor en el Modelo Pedagógico por competencias. Seminario en la Universidad Técnica Estatal de Quevedo. Quevedo: UTEQ.

Castro Cuesta, R. A. (2014). Análisis de las buenas prácticas del tutor universitario: estudio de caso de la Universidad Tecnológica de Chihuahua de México. Barcelona: Universidad Autónoma de Barcelona.

Coriat Benarroch, M., \& Sanz Oro, R. (2009). Orientación y tutoría universitaria. Granada: Universidad de Granada.

González Maura, V. (2006). El profesor tutor: una necesidad de la universidad del siglo XXI. Revista Cubana de Educación Superior Vol. 26 (2), (pp. 23-36).

González Tirados, R. M., \& González Maura, V. (2007). Investigación y desarrollo profesional en la universidad. Investigación y desarrollo profesional en la universidad. Revista Educación en Ingeniería, 2(4), 26-35.

Lara García, B. (2002). Una aproximación al concepto de tutoría académica en el Centro Universitario de Ciencias de la Salud. Investigación en Salud, vol. IV, núm. 1, abril. México, 12.

Lobato, C. (2004). La función tutorial universitaria: Estrategias de intervención. La Laguna: Servicio de Publicaciones de la Universidad de La Laguna.

Ojalvo Mitrany, V. (2005). Orientación y tutoría como estrategia para elevar la calidad de la educación. Revista cubana de educación superior, Vol. 25, № 2, 2005, págs. 3-18.

Ordaz Hernández, M. (2011). Concepción psicopedagógica del proceso de orientación: Estrategia para su implementación en la Universidad de Pinar del Río. Pinar del Río, Cuba. 


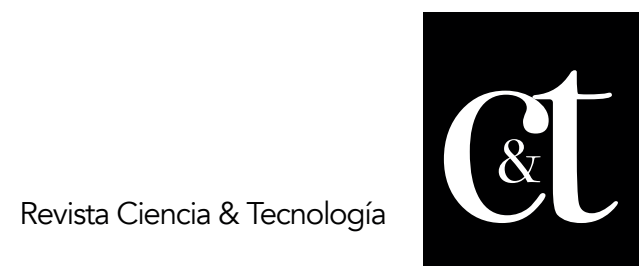

No. 19, 31 de julio de 2018

ISSN impreso: 1390 - 6321

Pi, M., Crespo A, Miranda Valdés M, González Jaime I, Caballero Graverán L, Crespo Morales A, y Blanco Ferrer M. (2011). El tutor Psicopedagógico. Una necesidad de la Universidad actual. Rev Dig Soc Inf [Internet]. 200

Real Academia de la Lengua Española (2007). Diccionario Manual de la Lengua Española. Barcelona: Larousse Editorial S. L.

Tejada Tayabas, J. M., \& Arias Galicia, L. F. (2006). El significado de tutoría académica en estudiantes de primer ingreso a licenciatura. En U. A. Potosí, San Luis de Potosí: División de Estudios de Posgrado, Facultad de Contaduría y Administración. 